\title{
Empowering Children's Critical Reflections on Al, Robotics and Other Intelligent Technologies
}

\author{
VICKY CHARISI \\ European Commission, Joint Research Centre (JRC), \\ Centre for Advanced Studies, Seville, Spain \\ vasiliki.charisi@ec.europa.eu \\ ELISA RUBEGNI \\ School of Computing \& Communications, InfoLab21, \\ Lancaster University, UK \\ e.rubegni@lancaster.ac.uk
}

\author{
LAURA MALINVERNI \\ University of Barcelona, Barcelona, Spain \\ malinverni.laura@gmail.com
}

MARIE-MONIQUE SCHAPER

Center for Computational Thinking and Design, Aarhus

University, Aarhus, Denmark

mmschaper@cc.au.dk

\begin{abstract}
The increasing presence of robotics, automated systems, and AI in everyday life is carrying important ethical and social implications for both those who design and develop them as well as for the users. Addressing these complex issues requires active collaboration between multiple stakeholders, including children. Nonetheless, even if emergent technologies are becoming increasingly present in education, most technology-mediated educational projects for children tend to focus on the development of technical skills, leaving little room for critical reflection. This tendency runs the risk of missing opportunities to truly empower children as critical users, (future) responsible designers and skilled stakeholders in the dialogue around ethical concerns on technology. Starting from this perspective, the workshop aims at tracing research lines and opening questions around strategies, methods, tools, and perspectives to support children in developing an ethical and critical sensitivity in the use, design, and development of emergent technologies.
\end{abstract}

\section{CCS CONCEPTS}

- Human-centered computing; • Human computer interaction (HCI);

\section{KEYWORDS}

Emergent technologies, Robotics, Artificial Intelligence, Automated Systems, Ethics, Children, Education, Policy-making

\section{INTRODUCTION}

As robots, automated systems and Artificial Intelligence (AI) evolve and gradually enter in everyday life, they are delineating novel techno-social realities which carry ethical and social concerns never seen before [15]. These issues are being actively explored by research in machine ethics [5], roboethics [14] and science, technology and society studies [3]. For instance, Riek \& Howard [10] pointed out the challenges, risks and opportunities related to therapeutic robots and the concerns about the lack of diversity in robots morphology and behaviour. Similarly, Sullins [12] highlighted the need for providing practitioners with practical guidance to understand ethical and legal issues in Human-Robot Interaction and listed open challenges for the design of ethical robots. In addition to this, books such as "Weapons of Math Destruction" [9] addressed the ethical issues related to Big Data and evidenced that inner biases and blind spots in algorithms can perpetuate or reinforce inequality. This broad panorama makes visible the increasing importance of an ethical perspective on emergent technologies and highlights relevant concerns related, for instance, to privacy, automated cars, dual-use of technology [12], attachment to social robots, lack of diversity in robot morphology and behaviour [10], reproduction of gender hierarchies [6] etc.

As several authors pointed out $[11,13,14]$, the complexity and ill-structured nature of these concerns requires for spaces and resources to promote an ethical and critical approaches to emergent technologies (i.e. robots, automated systems and AI) both for those who design them as well as for those who use them. While an increasing number of organizations such as IEEE [6] published works that tackle issues related to the use of robotics and AI in our everyday life, there is a lack of special attention on child users.

Only recently international organizations that focus on Child's Rights, such as UNICEF [12] and the United Nations' International Telecommunication Union, have started to examine the emerging ethical considerations regarding the use of AI, robotics and other intelligent technologies by children. The main purpose of such initiatives is to ensure that AI strategies, policies and ethical guidelines protect and uphold child rights. However, in order to address those complex issues and identify specific action points, constructive collaboration is needed among various stakeholders including 

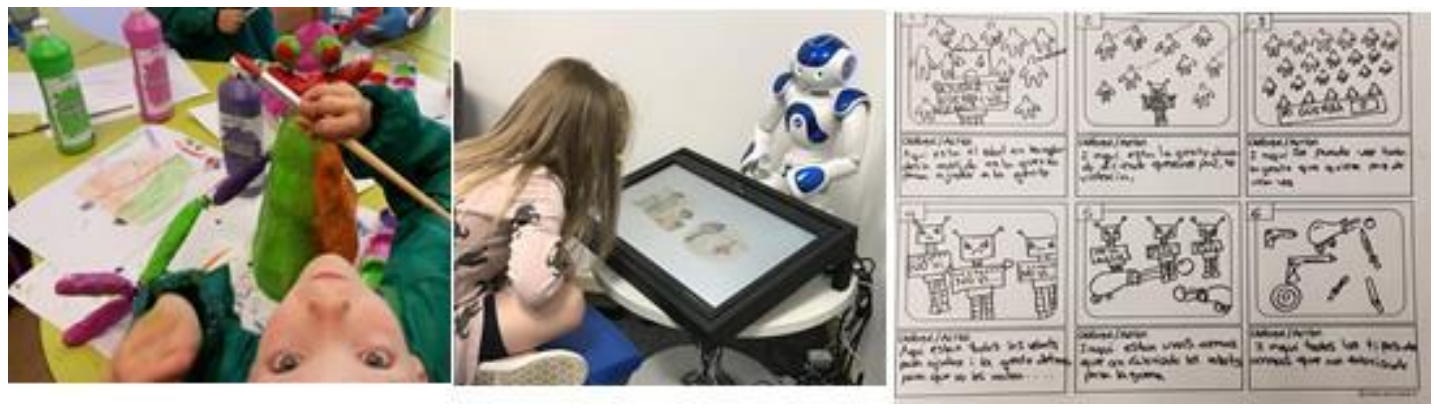

Figure 1: (1) Participatory Robot design session and concept identification with kindergarten children in a local school (Twente, the Netherlands); (2) A child interacting with Nao during a co-reading activity (Lincoln, UK); (3) storyboard describing the role of a robot president in society

researchers, developers, policy makers and end-users with a special focus on children.

This panorama points out the need for providing children with tools and strategies to be capable of dealing with the complexities of emergent technologies both as users as well as future designers/developers. Nonetheless, even if emergent technologies are becoming increasingly present in education [8] often, educational experiences with technology tend to focus mainly on learning of STEM-related concepts (e.g. physics, mathematics, etc.) or the development of skills such as teamwork, problem-solving and computational thinking $[1,2]$, leaving little or no room for critical reflection [10]. This tendency runs the risk of neglecting spaces for involving children in critically reflecting on the ethical and social impact of emergent technologies. Furthermore, it misses opportunities to actively involve children in constructive dialogues around these topics.

\subsection{Aim and Methodology}

Our aim is to trace research lines and opening questions around strategies, methods, tools, and perspectives to support childrenin developing an ethical and critical sensitivity in the use, design, and development of robots, automated systems and AI. The work- shop that we propose is part of a series of initiatives investigating this topic. We began exploring opportunities for children's critical reflections on AI, robotics and other intelligent technologies at a virtual workshop of the Interactional Conferences of Interaction Design and Children 2020 [4]. We invited researchers in this field to share their reflections up the following questions in a live discussion and through asynchronous communication channels beforeand after the workshop. In particular, the first workshop explored questions related to: "What are the current practices and policies of creating opportunities for children's reflections on AI, Robotics and other Intelligent Technologies?"; "How can we ensure that children are given the opportunities to critically reflect on the design and the use of AI, Robots and Intelligent Technologies?"; "What are the main ethical and social issues that deserve a particular attention when working with emergent technologies with children?"; "How can we address these issues? Which methods, techniques, approach, theories?"; "How to make these topics interesting, accessible and relevant for children?"; "What kind of tools and platforms we need in order to create a shared space for children's reflections?".
These questions were explored both in relation to the use of emergent technologies in education and play as well as in relation to the ways in which technology is taught in formal and informal contexts (e.g. educational robotics). We considered that addressing these questions could be helpful both to rethink the pedagogy of emergent technologies as well as to engage children as active participants in the discussion around the ethical and social issues of the technologies that they use $[5,7,9]$. These discussions provide a starting point towards the development of a holistic conceptual framework that focuses on (1) supporting children's critical reflections on intelligent technologies and (2) facilitating strategies to ensure that children's reflections are communicated to the different stakeholders involving the design of these technologies. Thus, by conducting this workshop series at different venues, we envision to bring together stakeholders with different research perspectives and professional interests.

\subsection{Organizers}

Vicky Charisi is a Senior Researcher at the Centre for Advanced Studies of the JRC, European Commission with a focus on the impact on AI on Human Behaviour, in particular on Child-Robot Interaction. Her research interests include child's cognitive and socio-emotional development and creative thinking in the context of emergent technologies, such as robotics, and her work informs policy-oriented discussions. Currently, she serves as a Chair of the IEEE Computational Intelligence Society for Cognitive and Developmental Systems TF for Human-Robot Interaction and she is a core member of the International Consortium for Socially Intelligent Robotics.

Laura Malinverni is a postdoctoral researcher at Universi de Barcelona. Her research focuses on creative methods to research and design emergent technologies with and for children. She holds a $\mathrm{PhD}$ in Information and Communication Technologies, a MSc in Cognitive Science and Interactive Media and a BA in Fine Arts. In the past, she has worked as researcher in both EU and nationalfunded projects as well as educator in several projects with at-risk youth.

Elisa Rubegni is a Lecturer in HCI and Children-Computer Interaction at Lancaster University, UK. Dr Rubegni research focuses on designing and evaluating children's experience in interacting with mobile, distributed technologies and social agents in educational 
contexts. Recently, she was awarded by Quality-Related Strategic Priorities Funding (QR-SPF) UKRC funding scheme to conduct a project focused on designing technology for very young children to empower them in reading at school. Dr. Rubegni is an editorial board member of the International Journal of Child-Computer Interaction. She is a constant presence at the IDC conference where she successfully organised and led three workshops.

Marie-Monique Schaper is a postdoctoral researcher at the Center for Computational Thinking and Design, Aarhus University. Her research focuses on mergingplayful technologies and design research in different disciplines of interaction. Marie is specifically interested in the exploration of novel strategies for participatory design methods and techniques to promote critical reflection around emergent technologies for children. She has worked in design projects with intergenerational stakeholders in the media industry and in academic contexts.

\subsection{Website}

Detailed information about the workshop can be found at: https: //sites.google.com/view/nordichi2020wsai/

\subsection{Pre-Workshop Plans}

We will use multiple recruitment approaches to attract participants to join the workshop. First, the organizers will use professional networks to contact researchers who are interested in participating in this workshop. Second, the organizers have access to research and professional mailing lists (e.g. British HCI, Human-Robot Interaction community) which will be used to advertise the workshop and recruit participants. Third, the organizers will invite the participants of the IDC workshop we carried out in June. Fourth, we will use social media channels to announce the workshop. Finally, the website will attract participants' attention and provide more details on the workshop.

\subsection{Workshop Structure}

The workshop's main aim is to allow researchers and practitioners to share their experiences, knowledge and opinions from their specific perspectives. We are planning to accommodate up to 20 papers. More authors of the same paper are welcome to participate, however just one person will present the position paper submitted.

To accommodate the workshop to the online format, we will ask participants to produce a short video (up to three minutes) with a summary of their paper that will be shared before the workshop together with the paper. This hand-out has the purpose to facilitate communication among them and begin the discussion and communication before the workshop.

The workshop will start with an initial presentation of the participants. After that, participants will be divided in small groups to facilitate discussion around key topics emerging from the submitted papers. Each small group discussion will be moderated by one of the workshop organizers. The discussion will be structured around a divergent-convergent approach, in which we will use previously designed personas to reflect on the values, positions and approaches of different stakeholders around key socio-technical issues related to intelligent technologies. Subsequently, the group activity will be presented to all participants and we will discuss on future paths and possibilities.

The workshop will be recorded using Zoom and we will ask participants to sign a consent form.

\subsection{Post-Workshop Plans}

After the workshop, we plan to disseminate outcomes of the discussion and reflections to a broader audience by editing a special issue in a journal. The journal will be selected after receiving the contribution and will be selected according to the specific contribution' types. We are planning to tackle journals such as: the International Journal of Children-Computer Interaction, or Behaviour \& Information Technology, or TOCHI, or the International Journal of Human-Computer Studies. In addition, we are planning a follow up workshop at the Joint Research Center of the European Commission and at other conference in related areas of HCI research.

\subsection{Call for Participation}

Robotics, automated systems, and AI are gradually shaping novel techno-social realities especially in educational contexts. In this context emerge important ethical and social implications for designers/developers and the users, especially children, however not enough space is given to a critical reflection on these issues.

This workshop aims to create a space to discuss social and ethical issues related to robotics, automated systems, and AI implied in educational environments. The main goal of the workshop is to bring together researcher/developers/practitioners who have interest in this topic and scaffold a collective discussion about these questions: "What are the current practices and policies of creating opportunities for children's reflections on AI, Robotics and other Intelligent Technologies?"; "How can we ensure that children are given the opportunities to critically reflect on the design and the use of AI, Robots and Intelligent Technologies?";" What are the main ethical and social issues that deserve a particular attention when working with emergent technologies with children?"; "How can we address these issues? Which methods, techniques, approach, theories?"; "How to make these topics interesting, accessible and relevant for children?";" What kind of tools and platforms we need in order to create a shared space for children's reflections?".

We are looking for highly motivated people who have experience in this field and wish to share their thoughts and knowledge in a highly interactive event. The workshop aims at providing participants a platform to increase their network as well as to develop innovative ideas, and learn something new.

Participants need to submit a position paper, 2-4 pages in the CHIExtendedAbstractsFormat, that outlines their view on the workshop's topic. We warmly encourage them to highlight the reasons for their interest in the topic including their previous work in the area. Paper submission will be done via easychair.

The workshop can accommodate approximately 20 participants. At least one author must register for the workshop and one day of the conference. In addition, the workshop participation willbe open to any NordiCHI participant, upon place availability. All attendants must register for both the workshop and one day of the 
conference. Authors will be selected by workshop organizers based on the papers' fit with the topic, originality, and quality. Intelligent provocative pieces are also welcome. Papers will be published on the website and shared with the participants.

The author of an accepted submission must attend the workshop, provide a short presentation, and engage in the three group work activities.

\subsection{Important Dates}

Submission deadline: 4th September 2020 23:59 PST. Participants notified of acceptance: (on or before) 6th September 2020. CameraReady Deadline (for workshop organizers): 7th September 2020 23:59 PST. Workshop day: 26th October 2020.

\section{REFERENCES}

[1] Saira Anwar and Nicholas Alexander Bascou. 2019. A Systematic Review of Studies on Educational Robotics. Journal of Pre-College Engineering Education Research (J-PEER) 9, 2.

[2] Fabiane Barreto Vavassori Benitti. 2012. Exploring the educational potential of robotics in schools: A systematic review. Computers and Education 58, 3: 978-988. http://doi.org/10.1016/j.compedu.2011.10.006

[3] V. Charisi, A. M. Alcorn, J. Kennedy, W. Johal, P. Baxter, and C. Kynigos. 2018 The near future of children's robotics. Proceedings of the 17th ACM Conference on Interaction Design and Children, 720-727.
[4] Vicky Charisi, Laura Malinverni, Elisa Rubegni, and Marie-Monique Schaper. 2020. Creating Opportunities for Children's Critical Reflections on AI, Robotics and Other Intelligent Technologies. International Conference of Interaction Design and Children, ACM, New York, NY, USA.

[5] Daniella DiPaola, Blakeley H. Payne, and Cynthia Breazeal. 2020. Decoding design agendas. Proceedings of the Interaction Design and Children Conference, ACM, 1-10. http://doi.org/10.1145/3392063.3394396

[6] IEEE Standards Association. 2018. The IEEE global initiative on ethics of autonomous and intelligent systems.

[7] Susan Lechelt, Yvonne Rogers, and Nicolai Marquardt. 2020. Coming to Your Senses: Promoting Critical Thinking about Sensors through Playful Interaction in Classrooms.

[8] R. Luckin. 2018. Machine Learning and Human Intelligence: The Future of Education for the 21st Century. UCL IOE Press. UCL Institute of Education, University of London, 20 Bedford Way, London WC1H 0AL.

[9] Marie-Monique Schaper, Laura Malinverni, and Cristina Valero. 2020. Robot Presidents: Who should rule the world? Teaching Critical Thinking in AI through Reflections upon Food Traditions. Proceedings of the 11th Nordic Conference on Human-Computer Interaction: Shaping Experiences, Shaping Society. http: //doi.org/doi.org/10.1145/3419249.3420085

[10] Rachel Charlotte Smith, Ole Sejer Iversen, and Mikkel Hjorth. 2015. Design thinking for digital fabrication in education. International Journal of Child-Computer Interaction 5: 20-28. http://doi.org/10.1016/j.ijcci.2015.10.002

[11] John P Sullins. 2015. Applied Professional Ethics for the Reluctant Roboticist. The Emerging Policy and Ethics of Human-Robot Interaction Workshop.

[12] United Nations Children's Fund (UNICEF). 2019. Workshop report: AI and child rights policy.

[13] Gianmarco Veruggio. 2006. The EURON Roboethics Roadmap. 612-617.

[14] Karolina Zawieska. 2020. Roboethics as a Research Puzzle. 2019 14th ACM/IEEE International Conference on Human-Robot Interaction (HRI), 731726: 612-613. 